\title{
A method for calculation of program package elements for singular clusters
}

\author{
Nikita Strelkovskii, Sergey Orlov
}

International Institute for Applied Systems Analysis

Lomonosov Moscow State University

"The problem of constructing optimal closed-loop control strategies under uncertainty is one of the key problems of the mathe matical control theory. Its solution would give a new impetus to the theory's development and create the foundation for its new applications" Arkady Kryazhimskiy (2013)

\section{Problem statement}

ET US CONSIDER A LINEAR DYNAMIC CONTROLLED SYSTEM, described by an ordinary differential equation

$$
\dot{x}(t)=A(t) x(t)+B(t) u(t)+c(t), t_{0} \leq t \leq \vartheta
$$

Open-loop control (program) $u(\cdot)$ is a measurable function on $\left[t_{0}, \vartheta\right], u(t) \in P \subset \mathbb{R}^{r}, P$ is a convex compact set. The initial state of the system maybe not known a priori: $x\left(t_{0}\right)=x_{0} \in$ $X_{0} \subset \mathbb{R}^{n}$, where $X_{0}$ is a finite known set. The terminal condition $x(\vartheta) \in M \subset \mathbb{R}^{n}$, where $M$ is a closed and convex set should hold.

A linear signal $y(t)=Q(t) x(t)$, where $Q(\cdot)$ is left piecewise continuous matrix-function, $Q(t) \in \mathbb{R}^{q \times n}, t \in\left[t_{0}, \vartheta\right]$, is observed by the controlling side.

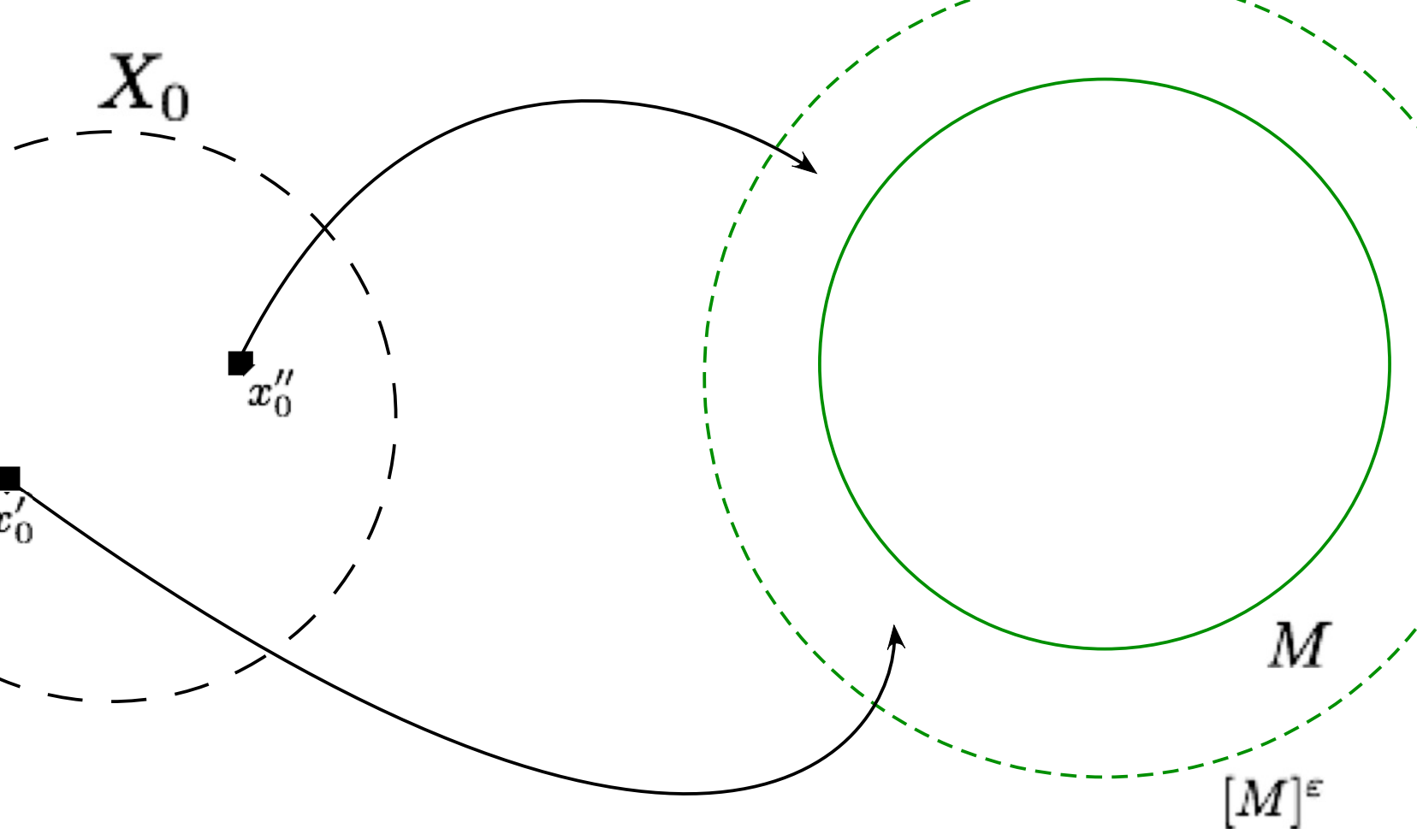

The problem of positional guidance is formulated as follows based on the given arbitrary $\varepsilon>0$ choose a closed-loop control strategy with memory, whatever the system's initial state $x_{0}$ from the set $X_{0}$, the system's motion $x(\cdot)$ corresponding to the chosen closed-loop strategy and starting at the time $t_{0}$ from the state $x_{0}$ reaches the state $x(\vartheta)$ belonging to the $\varepsilon$ neighbourhood of the target set $\mathrm{M}$ at the time $\vartheta$.

\section{Program packages method}

Homogeneous system, corresponding to (1)

$$
\dot{x}(t)=A(t) x(t)
$$

For each $x_{0} \in X_{0}$ its solution is given by the Cauchy formula: $x(t)=F\left(t, t_{0}\right) x_{0} ; F(t, s)\left(t, s \in\left[t_{0}, \vartheta\right]\right)$ is the fundamental matrix Homogeneous signal, corresponding to an admissible initial state $x_{0} \in X_{0}$ :

$$
g_{x_{0}}(t)=Q(t) F\left(t, t_{0}\right) x_{0}\left(t \in\left[t_{0}, \vartheta\right], x_{0} \in X_{0}\right) .
$$

Let $G=\left\{g_{x_{0}}(\cdot) \mid x_{0} \in X_{0}\right\}$ be the set of all homogeneous sig nals and let $X_{0}(\tau \mid g(\cdot))$ be the set of all admissible initial states $x_{0} \in X_{0}$, corresponding to the homogeneous signal $g(\cdot) \in G$ till time point $\tau \in\left[t_{0}, \vartheta\right]$ :

$$
X_{0}(\tau \mid g(\cdot))=\left\{x_{0} \in X_{0}:\left.g(\cdot)\right|_{\left[t_{0}, \tau\right]}=\left.g_{x_{0}}(\cdot)\right|_{\left[t_{0}, \tau\right]}\right\} .
$$

Program package is an open-loop controls family $\left.\left(u_{x_{0}} \cdot \cdot\right)\right)_{x_{0} \in X_{0}}$, satisfying non-anticipatory condition: for any homogeneous signal $g(\cdot)$, any time $\tau \in\left(t_{0}, \vartheta\right]$ and any admissible initial states $x_{0}^{\prime}, x_{0}^{\prime \prime} \in X_{0}(\tau \mid g(\cdot))$ the equality $u_{x^{\prime}}(t)=u_{x^{\prime \prime}}(t)$ holds for almost all $t \in\left[t_{0}, \tau\right]$.

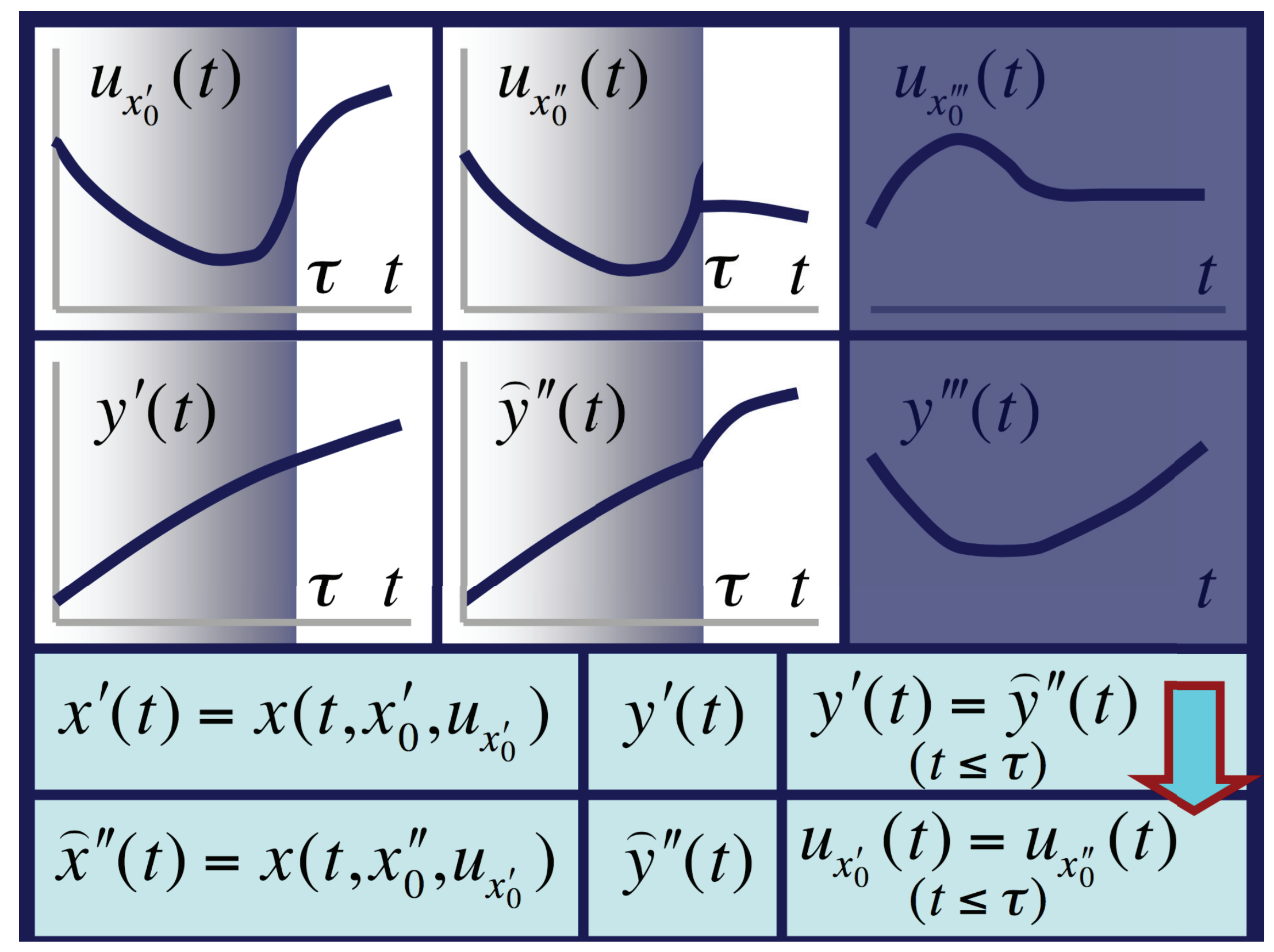

Program package $\left.\left(u_{x_{0}} \cdot \cdot\right)\right)_{x_{0} \in X_{0}}$ is guiding, if for all $x_{0} \in X_{0}$ holds $x\left(\vartheta \mid x_{0}, u_{x_{0}}(\cdot)\right) \in M$. Package guidance problem is solvable, if a guiding program package exists.

Theorem 1 ([1]). The problem of positional guidance is solvable if and only if the problem of package guidance is solvable.

\section{Algorithm for singular clusters}

An algorithm for the package guidance problem solution is proposed in paper [4], however, in some cases (so called singular clusters of the initial states set) it may not be applied, because the minimum condition degenerates. In this work an additional algorithm for tackling such cases is presented.

At a step $i=0,1, \ldots$ of the algorithm for arbitrary cluster $X_{0 r}\left(\tau_{k}\right) \in \mathcal{X}_{0}\left(\tau_{k}\right), r=1, \ldots, R\left(\tau_{k}\right), k=1, \ldots, K$ a non-empty set $w_{0}$ of indices is estimated $j, 1 \leq j \leq m$ such that to $j \in w_{0}$ corresponds non-empty set $\sigma_{j} \subset\left[\tau_{k-1}, \tau_{k}\right]$, such that

$$
\sum_{x_{0} \in X_{0 r}\left(\tau_{k}\right)} \sum_{q=1}^{n} D_{j q}(t) l_{x_{0_{q}}}^{*(i)} \equiv 0 \quad\left(t \in \sigma_{j}\right)
$$

The problem of estimation of the components $\left(u_{X_{0 r}\left(\tau_{k}\right)}^{*}(t)\right)_{j}$ of the guiding program package elements on the sets $\sigma_{j}, j \in w_{0}$ reduces to the following system of equations

$$
\left\{\begin{array}{l}
\sum_{j \in w_{0} \sigma_{j}} e_{j}(t) u_{j}(t) d t=v_{x_{0}}, \quad\left|u_{j}(t)\right| \leq a_{*} p \\
x_{0} \in X_{0 r}\left(\tau_{k}\right)
\end{array}\right.
$$

where

$$
\begin{aligned}
& \left.v_{x_{0}}=z_{x_{0}}-F\left(\vartheta, t_{0}\right) x_{0}+a_{*} p \sum_{k=1}^{K} \int_{\tau_{k-1}}^{\tau_{k}} \sum_{j=1}^{r} e_{j}(t) \operatorname{sign}\left(\sum_{\substack{\bar{x}_{0} \in X_{0_{0}}\left(\tau_{k} \\
r: x_{0} \in X_{0 r}\left(r_{k}\right)\right.}} \sum_{q=1}^{n} D_{j q}(t)\right)_{x_{0_{q}}}^{*}\right) d \tau, \\
& z_{x_{0}} \in \operatorname{Arg} \max _{z \in M}\left\langle z, l_{x_{0}}^{*}\right\rangle_{\mathbb{R}^{n}}, \\
& e(t)=\left(\begin{array}{c}
D(t)_{j 1} \\
\ldots \\
D(t)_{j n}
\end{array}\right), j=1, \ldots, m .
\end{aligned}
$$

Let us introduce functions $\lambda(\cdot,,,,,, \cdot):, \mathbb{R}^{n} \times \mathbb{R}^{n} \times[0,1] \times\left[\tau_{k-1}, \tau_{k}\right] \times \mathbb{N} \mapsto \mathbb{R}$ $\Lambda(\cdot, \cdot, \cdot, \cdot, \cdot): \mathbb{R}^{n} \times[0,1] \times\left[\tau_{k-1}, \tau_{k}\right] \times \mathbb{N} \times \mathbb{N} \mapsto \mathbb{R}:$

$$
\begin{aligned}
& \lambda\left(z_{x_{0}}, l_{x_{0}}, \mu, \sigma, w\right)=\sum_{x_{0} \in X_{0 r}\left(\tau_{k}\right)}\left\langle l_{x_{0}}, z_{x_{0}}\right\rangle_{\mathbb{R}^{n}}-\mu \sum_{j \in w} \int_{\sigma j}\left|\sum_{x_{0} \in X_{0 r}\left(\tau_{k}\right)} \sum_{q=1}^{n} D_{j q}(t) l_{x_{0_{q}}}\right| d t,
\end{aligned}
$$

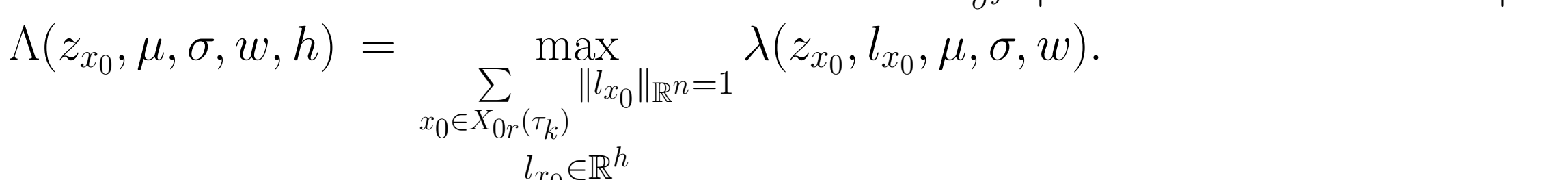

The input of the algorithm is

$$
\mu^{(0)}=1, \sigma_{j}^{(0)}=\sigma_{j}, w^{(0)}=w_{0}, z_{x_{0}}^{(0)}=v_{x_{0}} \quad\left(j \in w_{0}\right) .
$$

After the step $h-1, h=1,2, \ldots)$ of the algorithm the following is known

$$
\mu^{(h-1)}, w^{(h-1)}, \sigma_{j}^{(h-1)}, z_{x_{0}}^{(h-1)}, l_{x_{0}}^{*(1)}, \ldots, l_{x_{0}}^{*(h-1)},\left(u_{X_{0 r}\left(\tau_{k}\right)}^{*}(t)\right)_{j}\left(t \in \sigma_{j} / \sigma_{j}^{(h-1)}, j \in w_{0}\right)
$$

Let us describe the step $h, h=1,2, \ldots$ of the algorithm:

1. $\mu^{(h)}\left(0 \leq \mu^{(h)} \leq \mu^{(h-1)}\right)$ is calculated as the smallest root of the equation

$$
\Lambda\left(z_{x_{0}}^{(h-1)}, \mu, \sigma^{(h-1)}, w^{(h-1)}, n-h+1\right)=0 .
$$

2. If $\mu^{(h)}=0$, then $\left(u_{X_{0 .}\left(\tau_{k}\right)}^{*}(t)\right)_{j}=0, t \in \sigma_{j}^{(h-1)}, j \in w^{(h-1)}$ and the required element of the guiding program package is found. Otherwise the algorithm proceeds to the next step immediately.

3. The vector family $\left(l_{x_{0}}^{*(h)}\right)_{x_{0} \in X_{0}}$ is estimated such, that

$$
\sum_{x_{0} \in X_{0 r}\left(\tau_{k}\right)}\left\langle l_{x_{0}}^{*(h)}, l_{x_{0}}^{*(d)}\right\rangle=0
$$

for all $d=1, \ldots, h-1$ and

$$
\begin{aligned}
& \Lambda\left(z_{x_{0}}^{(h-1)}, \mu^{(h)}, \sigma^{(h-1)}, w^{(h-1)}, n-h+1\right)=
\end{aligned}
$$

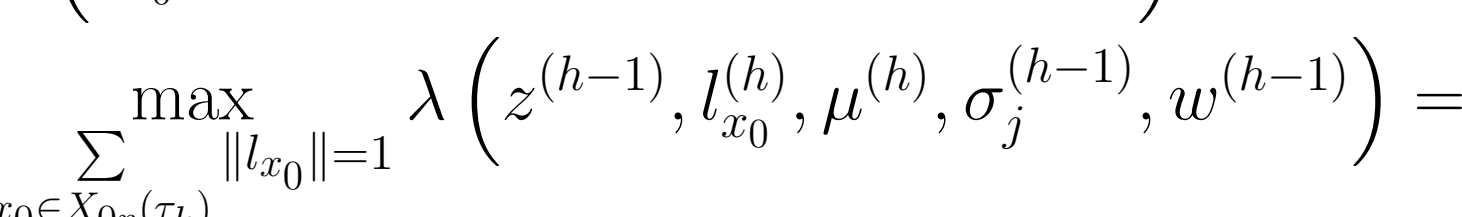

$$
\begin{aligned}
& \left.\sum_{\in X_{0 r}\left(\tau_{k}\right)}\left\langle l_{x_{0}}^{*(h)}, z_{x_{0}}^{(h-1)}\right\rangle-\mu^{(h)} \sum_{j \in w^{(h-1)}} \int_{\sigma h-1)} \mid \sum_{x_{0} \in X_{0 r}\left(\tau_{k}\right)} \sum_{q=1}^{n} D_{j q}(t)\right)_{x_{0_{q}}}^{*(h)} \mid=
\end{aligned}
$$

4. The set of indices $w^{(h)} \subset w^{(h-1)}$ is estimated and for each $j \in w^{(h)}$ a set $\sigma_{j}^{(h)} \subset \sigma_{k}^{(h-1)}, k \in w^{(h-1)}$ is found such that

$$
\sum_{x_{0} \in X_{0 r}\left(\tau_{k}\right)} \sum_{q=1}^{n} D_{j q}(t) l_{x_{0_{q 1}}}^{*(h)} \equiv 0 \quad\left(j \in w^{(h)}, t \in \sigma_{j}^{(h)}\right) .
$$

5. On the sets $\sigma_{k}^{(h-1)} / \sigma_{j}^{(h)}, k \in w^{(h-1)}, j \in w^{(h)}$ the components $\left(u_{X_{0 r}\left(\tau_{k}\right)}^{*}(t)\right)_{j}$ of the guiding program package elements are

$$
\left(u_{X_{0 r}\left(\tau_{k}\right)}^{*}(t)\right)_{j}=a_{*} p \operatorname{sign}\left(\sum_{x_{0} \in X_{0 r}\left(\tau_{k}\right)} \sum_{q=1}^{n} D_{j q}(t) l_{x_{0 q}}^{*(h)}\right) .
$$

6. If mes $\sigma^{(h)}=$ mes $\bigcup \sigma_{j}^{(h)}=0$, then $\left(u_{X_{0 r}\left(\tau_{k}\right)}^{*}(t)\right)_{j}$ is known on the whole set $\sigma=\bigcup \begin{aligned} & j \in w^{h} \\ & \sigma_{j}\end{aligned}$ and the problem is solved. Otherwise the algorithm proceeds to the next step

7. The following approximation is calculated

$$
\sum_{j \in w^{(h-1)}} \int_{\sigma_{j}^{(h-1)}} e_{j}(t) \operatorname{sign}\left(\sum_{\substack{\bar{x}_{0} \in X_{0}\left(\tau_{k}\right) \\ r: x_{0} \in X_{0 r}\left(\tau_{k}\right)}} \sum_{q=1}^{n} D_{j q}(t) l_{x_{0 q}}^{*(h)}\right) d t ;
$$

and the algorithm proceeds to the step $h+1$.

After $n \times m$ steps, in a generic case, $m$-dimensional control in $n$-dimensional problem is calculated on the whole half-interval $\left[\tau_{k-1}, \tau_{k}\right)$. Convergence of the algorithm follows from convergence of the corresponding algorithm in Euclidean space [3].

\section{Example}

Let us consider a linear control system

$$
\left\{\begin{array}{l}
\dot{x_{1}}=x_{2}, x_{1}(0)=x_{01} \\
\dot{x_{2}}=u, x_{2}(0)=x_{02}
\end{array}\right.
$$

on the time segment $[0,2]$.

$$
\begin{aligned}
M & =\left\{\left(\begin{array}{l}
x_{1}(t) \\
x_{2}(t)
\end{array}\right) \in \mathbb{R}^{2}:\left|x_{1}\right| \leq 1, x_{2} \in \mathbb{R}\right\} ; X_{0}=\left\{\left(\begin{array}{c}
\frac{3}{2} \\
0
\end{array}\right),\left(\begin{array}{c}
-\frac{3}{2} \\
0
\end{array}\right)\right\} \\
Q(t) & =\left\{\begin{array}{l}
(0,0), t \in[0,1] \\
(1,0), t \in(1,2]
\end{array} \quad u(t) \in P=\{u:|u| \leq 1\}, t \in[0,2] .\right.
\end{aligned}
$$

Since the solvability criterion holds, the package guidance problem is solvable. Using the algorithm from [4] the guiding program package elements for $x_{0}^{\prime}$ and $x_{0}^{\prime \prime}$ can be calculated. However, for the cluster $X_{0}=\left\{x_{0}^{\prime}, x_{0}^{\prime \prime}\right\}$ on the time interval $[0,1]$ there is an ambiguity in the minimum condition and the standard algorithm is not applicable. The presented method allows to reduce the problem to the system of two integral equations:

$$
\left\{\begin{array}{l}
x_{1}\left(2 \mid x_{0}^{\prime}\right)=\frac{3}{2}+\frac{1}{2} u_{X_{0}}(t)+\int_{1}^{2}\left(u_{X_{0}}(t)-t+1\right) d t=1 \\
x_{1}\left(2 \mid x_{0}^{\prime \prime}\right)=-\frac{3}{2}+\frac{1}{2} u_{X_{0}}(t)+\int_{1}^{2}\left(u_{X_{0}}(t)+t-1\right) d t=-1
\end{array}\right.
$$

Its solution gives that $u_{X_{0}}(t) \equiv 0, t \in[0,1]$.
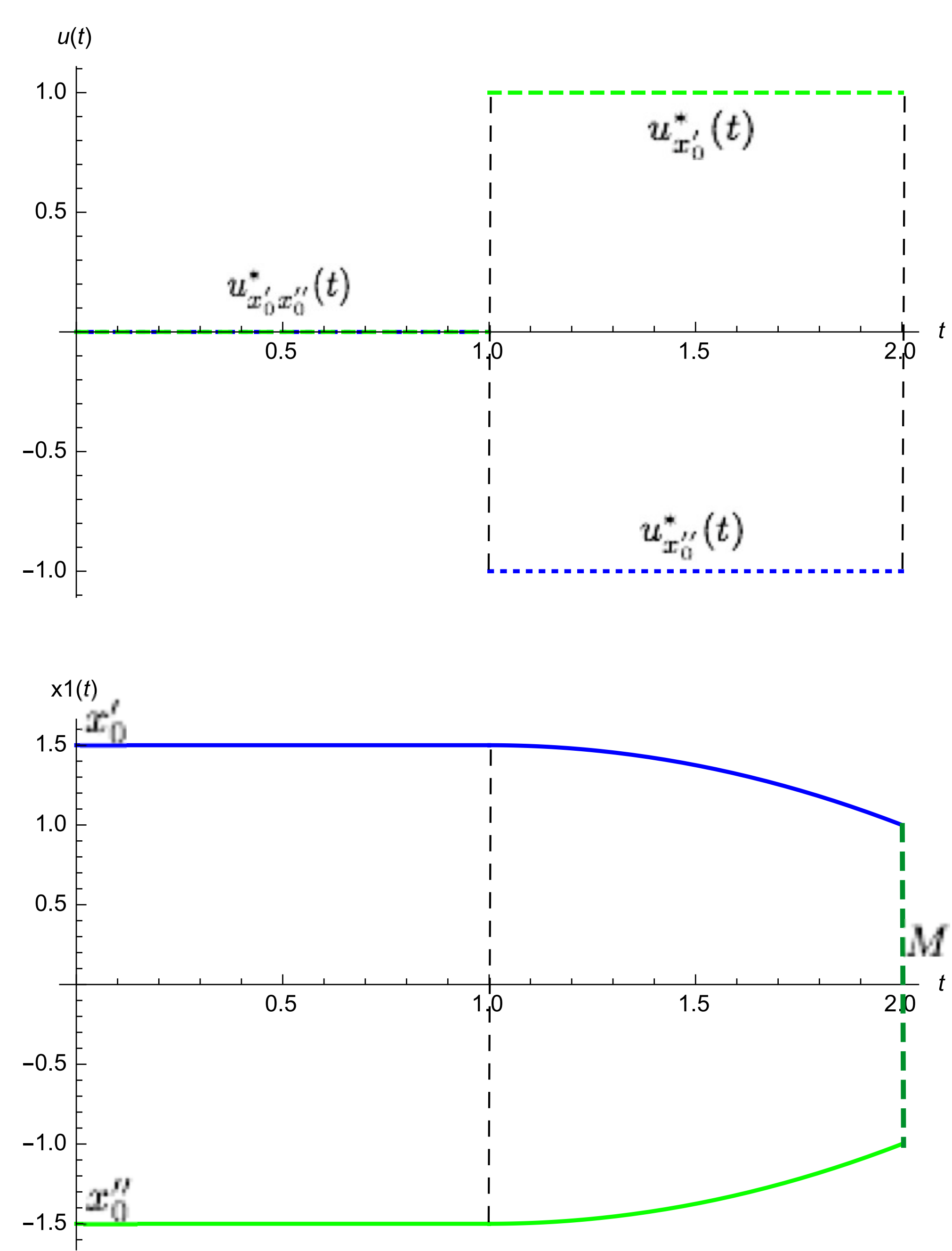

\section{References}

[1] Kryazhimskiy A.V., Osipov Yu.S. On the solvability of problems of guaranteeing control for partially observable linear dynamical systems // Proc. Steklov Inst. Math. 2012. No. 277. Pp. 144-159.

[2] Kryazhimskiy A.V., Strelkovskii N.V. An open-loop criterion for the solvability of a closed-loop guidance problem with incomplete information. Linear control systems // Trudy IMM UrO RAN. 2014. Vol. 20. No. 3. Pp. 132-147.

[3] Gindes V.B. Singular control in optimal systems // Izv. Vyssh. Uchebn. Zaved. Mat. 1967. No. 7. Pp. 34-42.

[4] Strelkovskii, N.V., Orlov, S.M. Moscow Univ. Comput. Math. Cybern. 2018. Vol. 42. No 2. Pp. 69-79. 